\title{
EVALUACIÓN DE LOS EXTRACTOS ETANÓLICOS DE LAS HOJAS DE MORA Y MANGO EN EL PROCESO DE DESHIDRATACIÓN DE CRUDOS PESADOS.
}

\section{Evaluation of ethanolic extracts of mulberry and mango leaves in the dehydration process of heavy crude oils.}

\author{
${ }^{1}$ Hernán Tixi Toapanta (iD), ${ }^{1}$ Natalia Barahona Alvear* iD , ${ }^{2}$ Henry Garmendia (iD \\ ${ }^{1}$ Escuela Superior Politécnica de Chimborazo, Facultad de Ciencias / Facultad de Mecánica, \\ Riobamba, Ecuador. \\ ${ }^{2}$ Universidad de los Andes, Facultad de Ingeniería, Laboratorio de Petróleo y Catálisis, Mérida, \\ Venezuela. \\ *natalia.barahona@espoch.edu.ec
}

$\mathrm{R}$ esumen

En esta investigación se evaluaron los extractos etanólicos de hojas de mora y mango, como potenciales deshidratadores de crudos pesados. Los extractos fueron obtenidos por maceración con una mezcla 70:30 \% vol. de etanol: agua. La efectividad fue determinada utilizando varias emulsiones acuosas, W/O, del 10 al 40\% en volumen, de los crudos pesados, tipo Hamaca y Jobo, provenientes de la Faja Petrolífera del Orinoco. Las emulsiones fueron dosificadas con 100, 500, 1000, 1500 y $2000 \mu \mathrm{L} / \mathrm{L}$ del extracto etanólico, agitadas, centrifugadas y separadas las fases. Los resultados mostraron que los desmulsificantes de mora y mango, poseen gran afinidad para deshidratar crudos pesados. El extracto de mango presento mayor eficiencia en romper emulsiones, W/O, del crudo Jobo, mientras que el extracto de mora para el crudo Hamaca.

Palabras claves: Emulsiones W/O, crudos pesados, extractos etanólicos, hojas de mora y mango.

\section{A bstract}

In this research, ethanolic extracts of mulberry and mango leaves were evaluated as potential dehydrators of heavy crude oils. The extracts were obtained by maceration with a 70:30 \% vol ethanol: water mixture. The effectiveness was determined using several aqueous emulsions, W/O, from 10 to $40 \%$ by volume, of heavy crudes, Hamaca and Jobo type, from the Orinoco Oil Belt. The emulsions were dosed with 100, 500,1000, 1000, 1500 and $2000 \mu \mathrm{L} / \mathrm{L}$ of the ethanolic extract, shaken, centrifuged and the phases separated. The results showed that the blackberry and mango demulsifiers have great affinity for dehydrating heavy crude oils. The mango extract presented greater efficiency in breaking emulsions, W/O, of Jobo crude oil, while the mulberry extract for Hamaca crude oil.

Keywords: Emulsion W/O, crude heavy oil, ethanol extract, mulberry leaves, mango leaves. 


\section{INTRODUCCIÓN}

Los crudos pesados y extrapesados constituyen la base de las reservas de hidrocarburos en Ecuador y otros países de Latinoamérica (1). Estos crudos producidos contienen algunas impurezas como agua, sal y sedimentos. En el proceso de extracción y producción de estos crudos se genera la formación de emulsiones no deseadas $(2,3)$. Estas emulsiones son del tipo $\mathrm{W} / \mathrm{O}$, es decir, agua en crudo y es un problema común en la industria petrolera (4). Además, estas emulsiones tienen una estabilidad que va desde unos minutos hasta años, dependiendo de la naturaleza del crudo y del contenido de agua. La solución a esto, es el uso de agentes químicos denominados desmulsificantes $(3,5,6)$.

A nivel de campo el tratamiento químico que logra el rompimiento de una emulsión en fases de aceite y agua se conoce como desemulsificación $(4,7,8,9)$. Desde el punto de vista del proceso, el productor de crudo está interesado en dos aspectos de la desemulsificación tales como, la velocidad de separación y la cantidad de agua remanente en el crudo después de la separación $(5,10,11)$. Para este último aspecto, normalmente no pueden contener más de $0.5 \%$ de agua y sedimentos (BS\&W) y $10 \mathrm{lb}$ de sal por mil barriles de crudo (PTB). Este bajo contenido de BS\&W y sal es requerido para reducir la corrosión y depósitos de sales (12).

El proceso de desemulsificación se da en dos pasos: la floculación y la coalescencia (13). Cualquiera de estos pasos puede determinar la velocidad del proceso de separación $(5,10,11)$.

Los aditivos químicos llamados desemulsificantes o rompedores de emulsión están diseñados para neutralizar el efecto emulsificante de los agentes que estabilizan la emulsión (14-17). En otras palabras, son compuestos de superficie activa, los cuales al ser agregados a la emulsión migran a la interfase crudo/agua, rompen o debilitan la película rígida y mejoran la coalescencia de las gotas de agua $(7,18,19)$.

Existen diferentes clases de desemulsionantes en el mercado, como los copolímeros de polioxie- tileno y polioxipropileno o las resinas de alquilfenol-formaldehído, las aminas alcoxiladas o sus mezclas $(8,20-22)$. Los esfuerzos actuales de investigación se centran en el desarrollo de desemulsionantes químicos rentables y respetuosos con el medio ambiente, caracterizados por una alta eficacia de separación del agua y una alta tasa de desemulsión, con el fin de cumplir con las especificaciones de las compañías petroleras (el corte de agua máximo permitido en el petróleo crudo suele ser del 0,1-0,5\%). En este contexto, el uso de extractos etanólicos de plantas para la desestabilización de emulsiones w/o es una opción muy prometedora.

Los extractos etanólicos de materia vegetal contienen los grupos principales de saponinas, taninos, aceites esenciales, compuestos órganosulfurados y flavonoides. Estos metabolitos secundarios se han utilizado en diferentes áreas: medicamentos, saborizantes, pigmentos y drogas recreativas $(9,23-26)$.

Los flavonoides y polifenoles presente en los extractos etanólicos de materia vegetal tienen propiedades desmulsificadoras. Dentro de estas, se encuentra las flavonas, isoflavonas y flavonoles. La presente investigación tiene como objetivo evaluar los extractos etanólicos de las hojas de mora y mango en el proceso de rompimiento de la emulsión W/O de los crudos pesados Hamaca y Jobo. Su efectividad representara una potencialidad en los procesos de deshidratación de crudos pesados y además tendrá un carácter ecológico y amigable al medio ambiente.

\section{MATERIALES Y MÉTODOS}

\section{Materiales}

La lista de materiales que se utilizaron para esta investigación incluía:

Crudos pesados Jobo y Hamaca (proveniente de la Faja Petrolífera del Orinoco, Venezuela), xyleno (Merck, 99\%), tolueno (Merck, 99\%), etanol (Merck, 99,5\%), agua destilada, hojas de mora y mango.

\section{Equipo}

Centrifuga (BenchMarks 2000, 2000 rpm máx) 


\section{Métodos}

\section{Obtención de los extractos etanólicos}

La Figura 1, presenta el diagrama de flujo del proceso de obtención, de los extractos etanólicos de mora y mango. Las hojas de mora y mango se recolectaron, y transportaron al laboratorio, para el proceso de maceración y obtención de los extractos. Se inició con un lavado de las hojas y su posterior secado, por 48 horas, en una estufa a $50{ }^{\circ} \mathrm{C}$.

Las hojas secas se molieron, y se procedió a macerarlas con una mezcla $70 \%$ etanol y $30 \%$ de agua. El tiempo de maceración fue de 10 días, con 2 agitaciones por día. Finalizada esta etapa, se procedió a separar a través de una membrana filtrante de $0.45 \mu \mathrm{m}$. El extracto etanólico obtenido se concentró en una unidad de destilación simple a $70^{\circ} \mathrm{C}$; se rotulo, para su evaluación posterior.

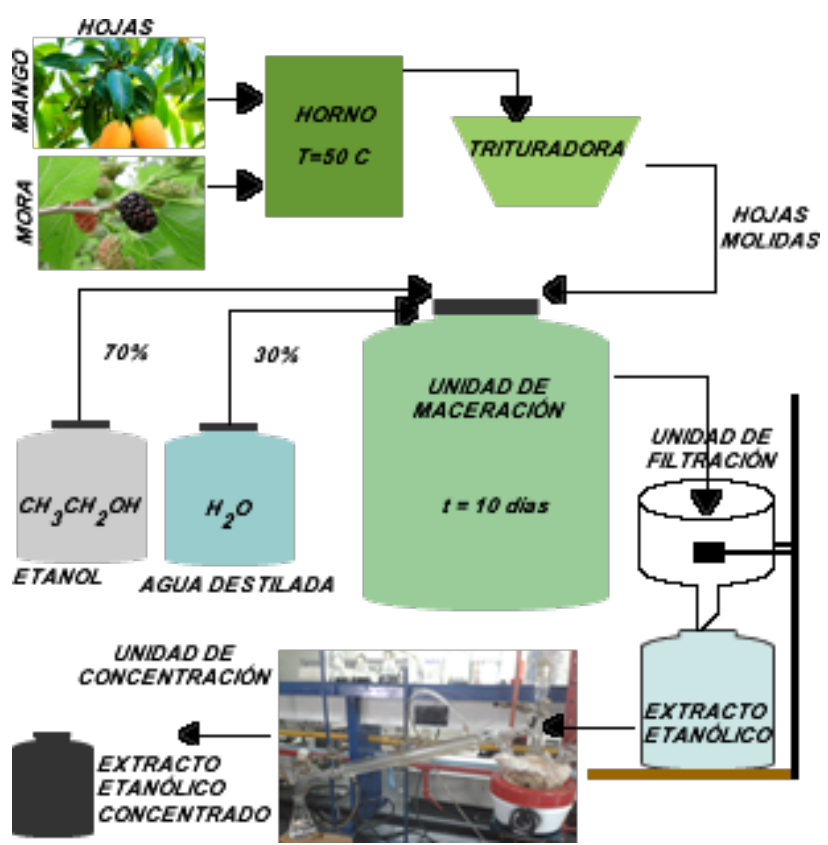

Figura 1. Diagrama del proceso de extracción etanólicos de hojas de mora y mango.

\section{Preparación de las emulsiones sintéticas}

Se prepararon emulsiones tipo $\mathrm{W} / \mathrm{O}$ para los crudos pesados Hamaca y Jobo. La Figura 2 muestra la unidad de preparación de las emulsiones. Las condiciones de operación de agitación son 2000 rpm y 1 h de mezclado.

La Tabla 1 muestra las emulsiones W/O formuladas y su rotulación.

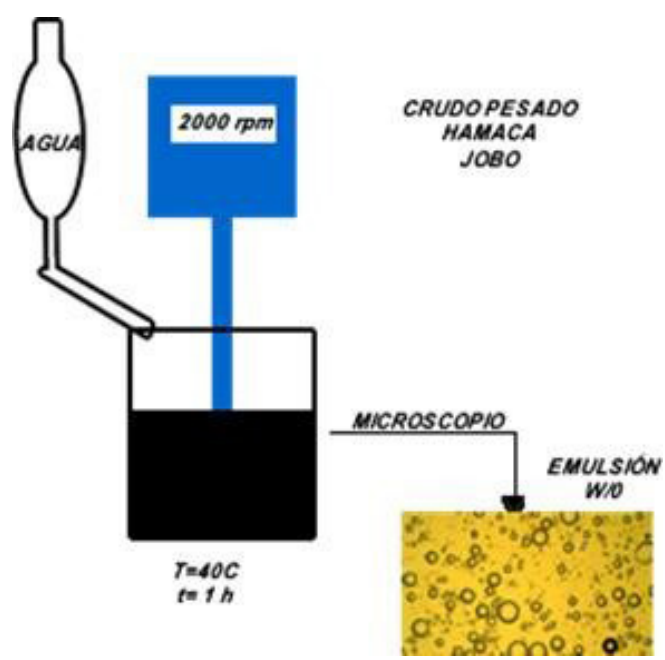

Figura 2. Proceso para la obtención de las emulsiones W/O.

\begin{tabular}{|l|c|c|c|c|}
\hline & \multicolumn{4}{|c|}{ Contenido de Agua } \\
\hline & $10 \% \mathrm{v}$ & $20 \% \mathrm{v}$ & $30 \% \mathrm{v}$ & $40 \% \mathrm{v}$ \\
\hline Emulsión(w/o) crudo Hamaca(EH) & EH10 & EH20 & EH30 & EH40 \\
\hline Emulsión(w/o) crudo Jobo(EJ) & EJ10 & EJ20 & EJ30 & EJ40 \\
\hline
\end{tabular}

Tabla 1. Emulsiones sintéticas W/O para los crudos Hamaca y Jobo.

\section{Eficiencia de desmulsificación}

El proceso de centrifugación permitió definir el contenido de agua y sedimentos de los crudos, siguiendo la norma ASTM D-4007. La eficiencia de separación de agua se calculó de la siguiente manera:

$\%$ Eficiencia $=\frac{\% \text { Agua separada }}{\% \text { Agua Emulsión }} \times 100$

\section{Preparación de los desmulsificantes}

La preparación de los diferentes desmulsificantes se realizó de la siguiente manera:

Se mide $9 \mathrm{ml}$ de solvente (tolueno o xileno) y se trasvasa a un balón aforado de $10 \mathrm{ml}$, luego se enrasa con $1 \mathrm{ml}$ de extracto de mora o mago. Se tapa el balón, se agita, hasta su homogenización y se rotula. La solución del desmulsificante fue al $10 \% \mathrm{v} / \mathrm{v}$.

\section{Ensayos de desmulsificación}

Para los ensayos de evaluación de los extractos desmulsificantes a base de hojas de mora y mango, se procedió de la siguiente manera:

Se homogeniza el crudo emulsionado a ensayar. Se coloca en el tubo de centrifugación $50 \%$ de crudo emulsionado y $50 \%$ de solvente xileno o tolueno y se dosifica 100,500, 1000, 1500 y 2000 $\mu \mathrm{L}$ de desmulsificante a evaluar en los tubos de centrifugación, se tapa y agita. Luego, se coloca los tubos en el baño María, $60{ }^{\circ} \mathrm{C}$ por $10 \mathrm{~min}$, 
y después se transfiere los tubos a la centrifuga, por $10 \mathrm{~min}$ y $1500 \mathrm{rpm}$. Por último, se registran las lecturas de los \% de volúmenes de agua y sedimentos, y el aspecto físico del agua que se separa de la emulsión, como turbia o clara, en las muestras.

\section{RESULTADOS Y DISCUSIÓN}

\section{Caracterización de los crudos pesados}

En la Tabla 2 se muestra la caracterización de los crudos pesados Hamaca y Jobo. Las propiedades de ${ }^{\circ}$ API y viscosidad los clasifica como crudos pesados y extrapesados.

\begin{tabular}{|c|c|c|}
\hline Propiedad & Valor & \\
\hline & Hamaca & Jobo \\
\hline${ }^{\mathbf{0}}$ API & 9,8 & 13,8 \\
\hline Carbón Conradson (\%peso) & 12,6 & 12,8 \\
\hline Contenido Asfalteno (\%peso) & 9,7 & 10,5 \\
\hline Azufre (\%peso) & 4,28 & 3,18 \\
\hline Contenido (V+Ni) ppm & 547 & 319 \\
\hline Viscosidad, cP & & \\
\hline @ $\mathbf{4 0} \mathbf{C}^{\mathbf{C}}$ & 8745 & 5439 \\
\hline @ $\mathbf{6 0}^{\mathbf{0}} \mathbf{C}$ & 2346 & 1280 \\
\hline
\end{tabular}

Tabla 2. Caracterización de los Crudos Pesados Hamaca y Jobo

\section{Efecto desmulsificante del extracto etanólico de mora}

A continuación, se muestran los resultados obtenidos de la dosificación del desmulsificante de mora en las diferentes emulsiones sintéticas W/O, de los crudos pesados Hamaca y Jobo.

\section{Caso crudo pesado Hamaca}

La Figura 3 muestra el efecto de la dosificación del desmulsificante extracto etanólico de mora, en el \% de eficiencia de separación del agua, presente en las diferentes emulsiones $\mathrm{W} / \mathrm{O}$, del crudo pesado Hamaca.

Se puede observar que a medida que incrementa la dosificación del desmulsificante, la eficiencia de separación de agua aumenta para todas las emulsiones $\mathrm{W} / \mathrm{O}$, estudiadas.

Las emulsiones EH10 y EH20, presentan un comportamiento parecido en la remoción del agua, con respecto a la variación de la dosificación del desmulsificante. Este hecho, puede deberse a que esta relación $\mathrm{W} / \mathrm{O}$, las gotas de agua están bastante mono dispersas y en un tamaño pequeño casi igual, que no permite romper la interfase $\mathrm{W} / \mathrm{O}$ en mayor proporción, para lograr una mayor eficacia de separación.

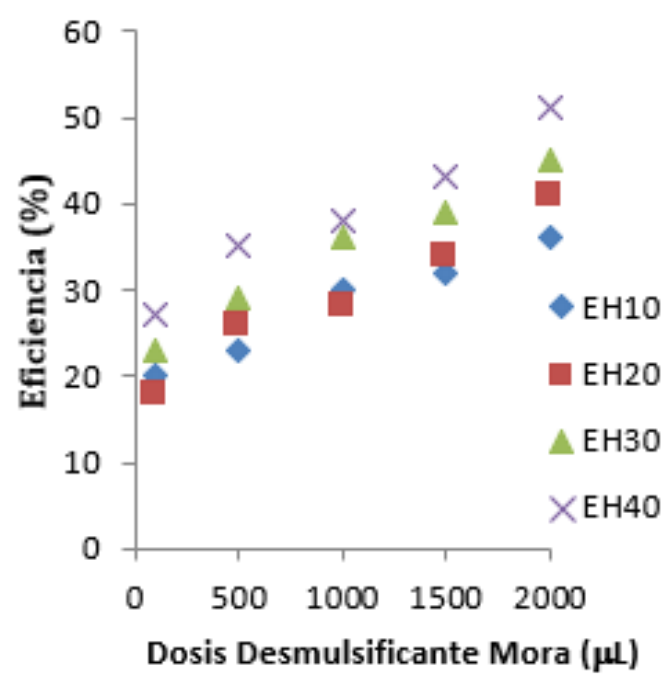

Figura 3. Efecto de la dosificación del desmulsificante de mora en función de la eficiencia de separación del agua en las diferentes emulsiones (W/O) del crudo pesado Hamaca

Mientras que para las emulsiones EH30 y EH40, se obtiene una mayor separación de agua, probablemente porque estas emulsiones contienen mayor polidispersidad de gotas, es decir, entre gotas grandes y pequeñas, que permite la adsorción del desmulsificante en la interfase $\mathrm{W} / \mathrm{O}$, facilitando mayor unión de gotas de agua y a su vez un incremento de la coalescencia del mismo.

Por lo tanto, a mayor contenido de agua en la emulsión la eficiencia de separación incrementa ya que disminuye la relación volumen de crudo en agua y el desmulsificante tiene una mayor ventaja de posicionarse en la interfase y reducir su interacción o romper la emulsión W/O, para separar el agua presente en la fase continua.

\section{Caso crudo pesado Jobo}

La Figura 4, muestra el efecto de la dosificación del demulsificante, extracto etanólico de mora, en el \% de eficiencia de separación del agua, presente en las diferentes emulsiones $\mathrm{W} / \mathrm{O}$ del crudo pesado Jobo.

Se puede observar que al aumentar la dosificación del demulsificante, extracto etanólico de mora, la eficiencia de separación de agua incrementa para todas las emulsiones $\mathrm{W} / \mathrm{O}$, estudiadas. 


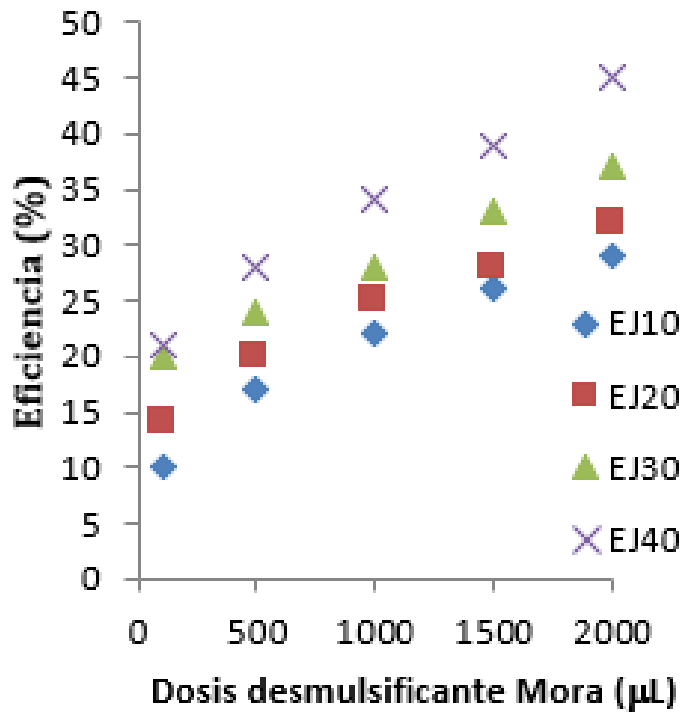

Figura 4. Efecto de la dosificación del desmulsificante de mora en función de la eficiencia de separación de agua en las diferentes emulsiones (W/O) del crudo pesado Jobo.

Las emulsiones EJ10, EJ20 y EJ30 tienen un comportamiento similar, esto es, que a medida que aumenta el contenido acuoso en la emulsión, la separación se incrementa; pero si se compara el incremento de agua separada resulta que es muy cercana, este hecho puede deberse a que la emulsión W/O quedo más mono dispersa que no permite una mayor interacción gota a gota, y el desmulsificante no logra posicionarse en la interfase $\mathrm{W} / \mathrm{O}$, que permita un mayor rompimiento de la emulsión; mientras que la emulsión EJ40 por su alto contenido de agua y posiblemente presentar una mayor polidispersidad en tamaño de gotas de agua, permite que el demulsificante se coloque en la interfase y mejore el rompimiento de la emulsión.

Por consiguiente, se logró el rompimiento de la emulsión W/O del crudo Jobo, y es de notar que el agua separada fue clara.

\section{Efecto desmulsificante del extracto etanólico de mango}

\section{Caso crudo pesado Hamaca}

La Figura 5, muestra el comportamiento de la dosificación del demulsificante, extracto de mango, en el rompimiento de las emulsiones W/O del crudo Hamaca. Se puede observar que a medida que aumenta la dosis del desmulsificante la eficiencia de separación del agua se incrementa.

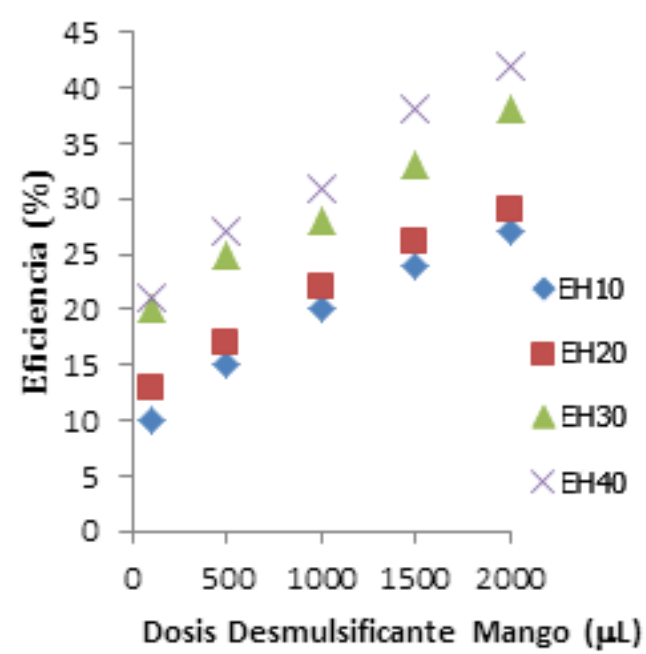

Figura 5. Efecto de la dosificación del desmulsificante de mango en función de la eficiencia de separación de agua en las diferentes emulsiones (W/O) del crudo pesado Hamaca.

Las emulsiones EH10 y EH20 presentan un comportamiento análogo en el rompimiento de la emulsión, posiblemente debido a que las gotas de aguas son de tamaño pequeño y monodispersas, lo cual hace que disminuya la interacción entre gotas. El desmulsificante disminuye su actividad al no inhibir o eliminar las fuerzas de presión que se ejerce en la interfase agua-crudo, resultando en un bajo rompimiento de la emulsión W/O.

Por el contario, observando las emulsiones EH30 y EH40 su comportamiento de desmulsificación son parecidas, esto es debido al alto contenido de agua. La distribución de gotas está polidispersa, mejorando la interacción entre gotas y el extracto de mango que logra una mayor efectividad en el rompimiento de dichas emulsiones.

\section{Caso crudo pesado Jobo}

La Figura 6, muestra el comportamiento lineal de la dosis del desmulsificante, extracto de mango, para las diferentes emulsiones W/O del crudo pesado Jobo, en función de la eficiencia de separación de agua. Se observa que a medida que aumenta la dosificación del desmulsificante, la eficiencia de separación del agua también incrementa. Las emulsiones EJ10, EJ20 y EJ30 presenta un comportamiento semejante en la separación del contenido de agua, quizá se deba a que el contenido de agua en la emulsión presenta cierta monodispersión de gotas que hace que la interacción entre gotas se minimice y el desmulsificante reduzca su acción de romper dichas emulsiones.

Mientras que, la emulsión EJ40 muestra un com- 
portamiento diferente, que puede deberse a que, el contenido de agua en la emulsión W/O presenta una polidispersidad de las gotas de agua, que resulta en mejor interacción entre ellas. El agente desmulsificante mejora su eficacia de separación ya que, logra posicionarse en la interface agua-crudo y reducir o eliminar esta para efectuar la coalescencia del agua.

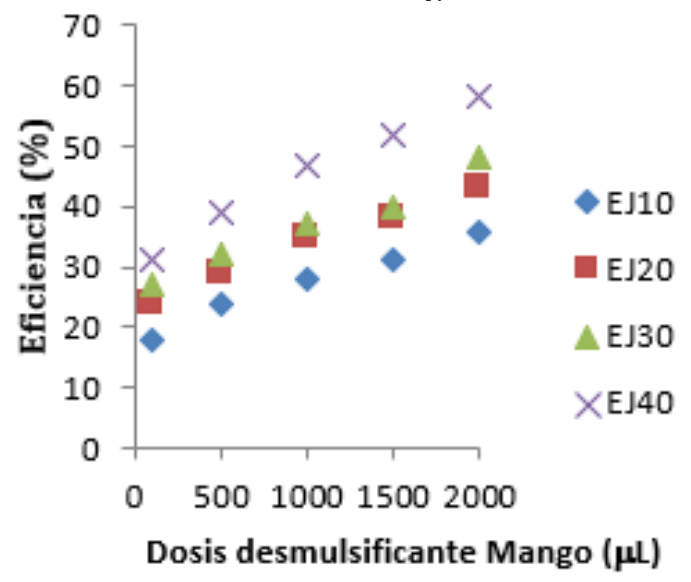

Figura 6. Efecto de la dosificación del desmulsificante de mango en función de la eficiencia de separación de agua en las diferentes emulsiones $(\mathrm{W} / \mathrm{O})$ del crudo pesado Jobo.

Evaluación comparativa de los extractos etanólicos de mora y mango

Para clarificar el efecto del tamaño de la gota en el grado de estabilidad de la emulsión, se tomó en consideración la Figura 7, dónde se muestra la estabilidad o inestabilidad de una emulsión $\mathrm{W} / \mathrm{O}$, en función de la igualdad en el tamaño de la gota. Si la emulsión es estable, los demulsificantes ejercerán poca fuerza para romper la emulsión, mientras que si los tamaños de gotas en la emulsión W/O son variables, genera mayor interacción entre ellas, lo cual mejora la efectividad del desmulsificante.

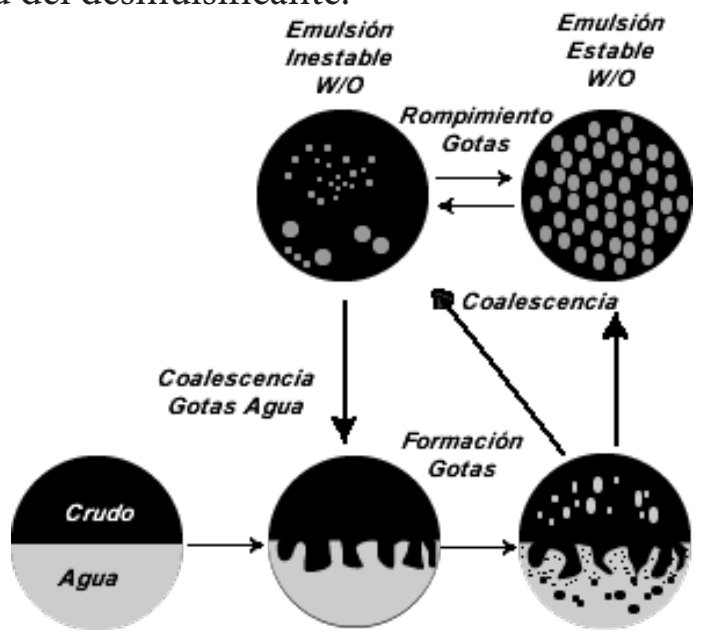

Figura 7. Esquema del efecto que tiene el tamaño de gota en las emulsiones W/O.
Las Figuras 8 a 11 muestran los resultados obtenidos al comparar las diferentes dosificaciones extractos etanólicos de mora y mango en la efectividad de separación del contenido de agua presente en las diferentes emulsiones W/O para los dos crudos pesados Hamaca y Jobo.

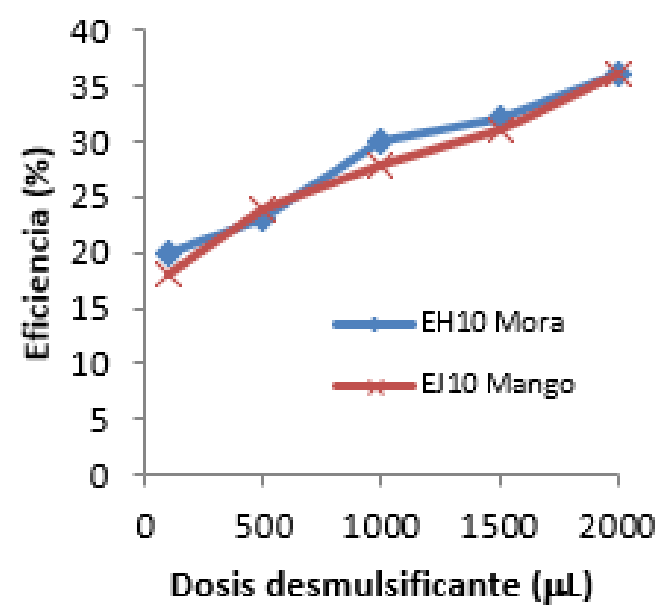

Figura 8. Efecto de la dosificación del desmulsificante de mora y mango en función de la eficiencia de separación del agua para la emulsión (W/O) 10:90 \%vol., en crudos Hamaca y Jobo.

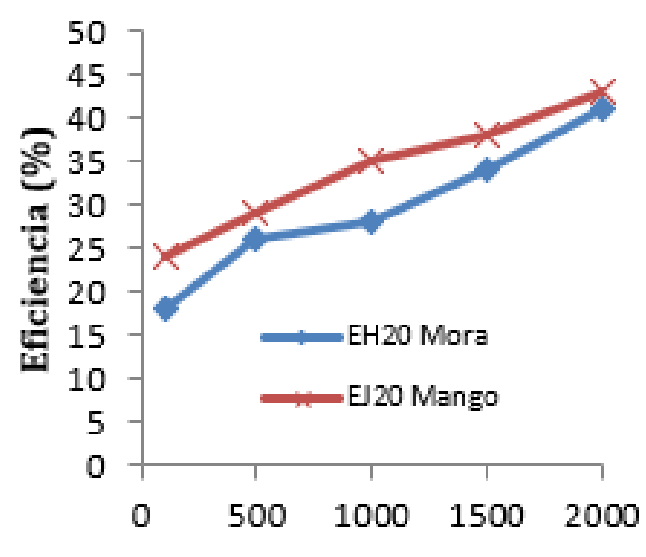

Dosis desmulsificante $(\mu \mathrm{L})$

Figura 9. Efecto de la dosificación del desmulsificante de mora y mango en función de la eficiencia de separación del agua para la emulsión W/O, 20:80 \%vol., en crudos Hamaca y Jobo.

Se observa que el desmulsificante de mora y mango presenta un comportamiento semejante en la eficiencia de separación del contenido de agua como se muestra en las Figuras 8 y 10.

Mientras que, en las Figuras 9 y 11 se observa que el extracto de mango tiene una mayor eficiencia de separación del contenido de agua en el crudo pesado Jobo con respecto al crudo Hamaca. 


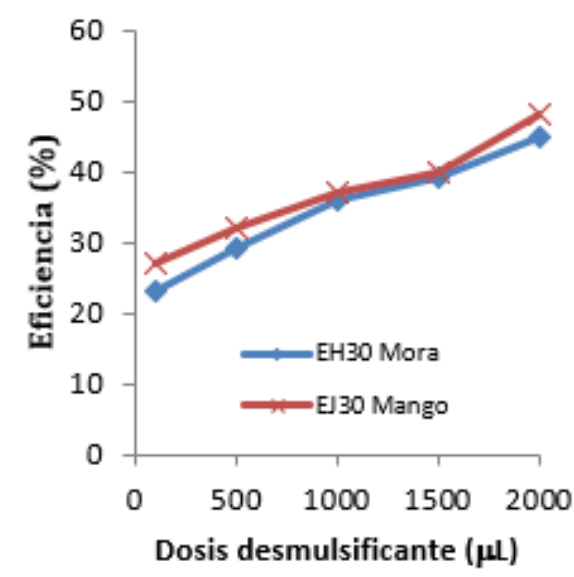

Figura 10. Efecto de la dosificación del desmulsificante de Mora y Mango en función de la eficiencia de separación del agua para la emulsión W/O, 30:70 \%vol, en crudos Hamaca y Jobo.

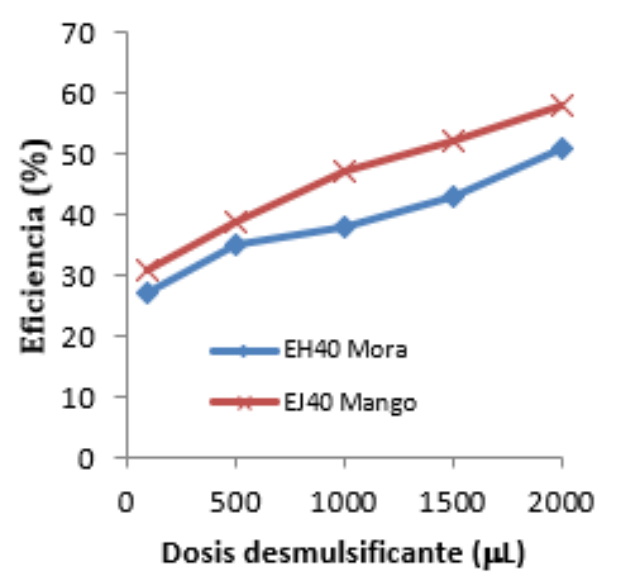

Figura 11. Efecto de la dosificación del desmulsificante de mora y mango en función de la eficiencia de separación de agua para la emulsión (W/O) 40:60 \%vol. del agua, en crudos Hamaca y Jobo.

\section{Hamaca.}

La separación de agua en el crudo pesado Jobo fue limpia, mientras que, en el crudo hamaca fue turbia.

La eficiencia de separación de agua se ve favorecida para los desmulsificante de mora y mango a altos contenidos de agua emulsionada en el crudo pesado.

Los formulados de mora y mango, tiene una gran potencialidad, en deshidratar crudos pesados con altos contenidos de agua emulsionada y además, son formulados ecológicos y con bajo impacto ambiental.

$\mathrm{R}$ eferencias

1. González C, Santiago J. Obtención y Caracterización de Fracciones SARA de Crudos Ecuatorianos de Diferente Procedencia. 2019; Tesis de Pregrado: Universidad de las Fuerzas Armadas.

2. Requeijo D, Ochoa A. Nuestro Petróleo a tu alcance. Editorial Biosfera. 2009.

3. Al-Sahhaff TA, Fahim MA, Elsharkawy AM. Effect to inorganic solids, wax to asphaltene ratio and water cut on the stability of water-in-crude oil emulsions. J. Dis. Sci. Technol. 2009, 30: 597-604.

4. Abdurahman, H, Mohd, A, \& Rosli, M. Charcterization and demulsification of water-in crude oil emulsions. Journal of Applied Sciences. 2017, 1437-1441.

5. Noboa, G., Márquez, L., \& López, J. Tamaño de gota: Factor determinante sobre la velocidad de clarificación de una emulsión o/w. Ciencia e Ingeniería. 2017, 259-264.

6. Temple-Heald C, Davis C, Wilson N y Readman N. Developing new surfact chemistry for breaking emulsions in heavy oil. J. Pet. Technol. 2015, 66: 30-36.

7. Velazque I, Pereira J. Emulsiones de agua en crudo. Aspectos Generales. Revista de Ingeniería UC, 2014, Vol. 21, No. 3, pp. 45-54.

8. Dos Santos R, Bannwart A, y col. Physico-chemical properties of heavy crude oil-inwater emulsions stabilized by mixtures of ionic and non-ionic ethoxylated nonylphenol surfactants and medium chain alcohols. Chemical Engineering Research and Design, 2011, 89, 957-967.

9. Aruna GR, Geetha MY y Manjunath G. Chemical composition and pharmacological functions and principles of mulberry: A Review. International Journal of Applied Research, 2017,3(4): 251-254.

10. Noboa, G, Márquez, L, \& López, J. Tamaño de gota: Factor determinante sobre la velocidad de clarificación de una emulsión o/w. Ciencia e Ingeniería. 2017, 259-264. 
11. Ahmed M, Notaila, M. and Tahany M. Investigation of Kinetic and Rheological Properties for the Demulsification Process, 2013, 22, 117-127.

12. State, E. Formulation and production of Crude Oil Demulsifiers From. 2010, 2, 26-37.

13. Temple-Heald C, Davis C, Wilson N y Readman N. Developing new surfact chemistry for breaking emulsions in heavy oil. J. Pet. Technol. 2015, 66: 30-36.

14. Azizi, K. and Nikazar, M., Characterization of chemical demulsification of oil in water emulsion: comparison between a kinetics model and laboratory experiments. Petroleum Science and Technology, 2015, 33, 8-15.

15. Cendejas, G, Arreguín, F, Castro, LV, Flores, EA and Vazquez F. Demulsifying super-heavy crude oil with bifunctionalized block copolymers. Fuel, 2013,103, 356.

16. Velazque I, Pereira J. Emulsiones de agua en crudo. Aspectos Generales. Revista de Ingeniería UC, 2014, Vol. 21, No. 3, pp. 45-54.

17. Issaka, SA, Nour, AH and Yunus, RM. Review on the fundamental aspects of petroleum oil emulsions and techniques of demulsification. Journal of Petroleum \& Environmental Biotechnology, 2015, 6, 214.

18. Karcher V, Perrechil, FA and Bannwart, AC. Interfacial energy during the emulsification of waterin-heavy crude oil emulsions. Brazilian Journal of Chemical Engineering, 2015, 32, 127.

19. Peña, AA, Hirasaki, GJ and Miller, CA. Chemically induced destabilization of water-in-crude oil emulsions. Industrial \& Engineering Chemistry Research, 2005, 44, 1139.

20. Dos Santos R, Bannwart A, y col. Physico-chemical properties of heavy crude oil-in water emulsions stabilized by mixtures of ionic and non-ionic ethoxylated nonylphenol surfactants and medium chain alcohols. Chemical Engineering Research and Design, 2011, 89, 957-967.

21. Silva, EB, Santos, D, Alves, DM, Barbosa, MS, Guimarães, RC, Ferreira, BM, Guarnieri, RA, Franceschi, E, Dariva, C, Santos, AF and Fortuny, M. Demulsification of heavy crude oil emulsions using ionic liquids. Energy \& Fuels, 2013, 27, 6311.

22. Acevedo, S., Gutierrez, X., Rivas, H. Bitumen-in-water emulsions stabilized with natural surfactants. J. Colloid Interface Sci. 2001,242, 230-238.

23. Britt M. Burton-Freeman, Amandeep K. Sandhu, Indika Edirisinghe. Mangos and their bioactive components: Adding variety to the fruit plate of health. Food\&Function, 2017, May, 1, 55.

24. Wan-Taek J, O-Chul K, y col. Qualitative and quantitative analysis of flavonoids from 12 species of Korean mulberry leaves. 2018. 55(5): 1789-1796.

25. Aruna GR, Geetha MY y Manjunath G. Chemical composition and pharmacological functions and principles of mulberry: A Review. International Journal of Applied Research, 2017, 3(4): 251-254.

26. Sánchez José, Moreno D, Álvarez D y col. Phenolic profile, antioxidant and anti-proliferative activities of methanolic extracts from Asclepias linaria Cav. Leaves, Molecules 2020, 25, 54. 